\title{
Dermatite associada à incontinência na população idosa: uma revisão integrativa
}

\author{
Dayse C. Nascimento, ${ }_{1}^{1}$ Clícia V. Cunha, ${ }^{2}$ Lucia Helena G. Penna, ${ }^{3}$ Norma Valéria D. O. Souza, ${ }^{4}$ Graciete S. \\ Marques $^{5}$
}

\section{Resumo}

A dermatite associada à incontinência (DAI) é uma lesão cutânea potencialmente grave que acomete grande parte da população idosa com algum tipo de incontinência, ocasionando importante impacto social e hospitalar. O presente artigo trata-se de uma revisão integrativa cujo objetivo é analisar produções científicas que abordam esse tipo de lesão considerando seus conceitos e medidas de prevenção e tratamentos propostos. Foi realizado levantamento bibliográfico manual e eletrônico nas bases de dados da Biblioteca Virtual de Saúde (BVS) a partir das associações dos seguintes descritores: 1) dermatite, incontinência urinária/fecal, idosos; 2) dermatite, incontinência, idosos, enfermagem; com seleção de 15 artigos publicados em texto completo no período de 2006 a 2015. Os resultados apontaram a DAI como um problema de saude social e hospitalar e apresentaram as iniciativas de investigação relacionadas à prevenção e tratamento. Conclui-se que ainda há uma carência em recomendações baseadas em evidências que visem a um acompanhamento individualizado e minucioso com registros adequados, de forma a facilitar a identificação, a diferenciação e o manejo da DAI.

Descritores: Dermatite; Incontinência urinária/fecal; Idosos; Enfermagem.

\section{Abstract \\ Dermatitis Associated with Incontinence in the elderly: an integrative review}

Dermatitis associated with incontinence (DAI) is a potentially serious skin lesion that affects much of the elderly population with some form of incontinence causing major social impact and at the hospital as well. This article is an integrative review aimed at analyzing scientific publications that address this type of injury considering its concepts and proposed prevention and treatment measures. It was conducted a manual and an electronic research on the Virtual Health Library databases (BVS) with the following descriptors associations:1) dermatitis, urinary incontinence/fecal, seniors; 2) dermatitis, incontinence, senior; with a selection of 15 articles published in full text from 2006 to 2015. The results showed DAI as a social and a hospital health problem and presented research initiatives related to its prevention and treatment. It is concluded that there is still a lack of evidence-based recommendations aimed at an individual and thorough follow up with proper records in order to facilitate the identification, differentiation and management of DAI.
1. Comissão de Curativos. Hospital Universitário Pedro Ernesto. Rio de Janeiro, RJ, Brasil.

2. Seção Enfermagem Mulher e Criança. Hospital Universitário Pedro Ernesto. Rio de Janeiro, RJ, Brasil.

3. Departamento de Enfermagem Materno-Infantil. Faculdade de Enfermagem. Univer-sidade do Estado do Rio de Janeiro. Rio de Janeiro, RJ, Brasil.

4. Departamento de Enfermagem Médico-Cirúrgica. Faculdade de Enfermagem. Universidade do Estado do Rio de Janeiro. Rio de Janeiro, RJ, Brasil.

*Endereço para correspondência: Coordenação de Enfermagem, HUPE Boulevard 28 de Setembro, 77 3 andar Rio de Janeiro, RJ. CEP: 20551-030.

E-mail: daysecnascimento@hotmail.com

Revista HUPE, Rio de Janeiro, 2016;15(1):37-42

doi: $10.12957 /$ rhupe.2016.22840

Recebido em 28/03/2016. Aprovado em 03/05/2016.

Keywords: Dermatitis; Urinary/Fecal incontinence; Seniors; Nursing.

\section{Resumen}

\section{Dermatitis asociada con la incontinencia en los ancianos: una revisión sistemática}

La dermatitis asociada con la incontinencia (DAI) es una lesión de la piel potencialmente grave que afecta a gran parte de la población de edad avanzada con un cierto grado de incontinencia, causando gran impacto social y hospitalario. Por consiguiente, este artículo presenta una revisión integradora cuyo objetivo es analizar las publicaciones científicas que se ocupan de este tipo de lesión, teniendo en cuenta sus conceptos, medidas de prevención y tratamientos propuestos. Se efectuó la revisión manual y electrónica de la literatura, en la base de datos de la Biblioteca Virtual en Salud (BVS), de las asociaciones de palabras clave: 1) dermatitis, incontinencia urinaria/fecal, tercera edad; 2) dermatitis, incontinencia, tercera edad, enfermería, con una selección de 15 artículos publicados en texto completo en el periodo de 2006-2015. Los resultados mostraron la dermatitis asociada con la incontinencia (DAI) como un problema social y de salud hospitalaria y demostraron iniciativas de investigación relacionadas con la prevención y el tratamiento. Se llegó 


\section{Artigo original}

a la conclusión de que todavía faltan pruebas basadas en recomendaciones dirigidas a un seguimiento individual y exhaustivo con los registros adecuados, con el fin de facilitar

\section{Introdução}

Nos últimos anos, o Brasil vem apresentando um novo padrão demográfico que se caracteriza pela redução da taxa de crescimento populacional e por transformações profundas na composição de sua estrutura etária, com um significativo aumento do contingente de idosos. De acordo com dados do Instituto Brasileiro de Geografia e Estatística (IBGE), o índice de brasileiros acima de 60 anos vem aumentando progressivamente, promovendo uma mudança na estrutura populacional e aumentando a necessidade de direcionamento dos cuidados a esses indivíduos, a fim de assisti-los em todos os níveis de complexidade. ${ }^{1}$

Destaca-se que as mudanças provenientes do processo de envelhecimento resultam em decréscimo na capacidade fisiológica e diminuição de resposta do organismo a determinados eventos estressantes. Assim, ocorrem alterações celulares, teciduais, anatômicas e funcionais nos diferentes sistemas destes indivíduos.

No entanto, um dos sistemas visivelmente mais acometido é o sistema tegumentar. Sabe-se que a pele constitui um dos órgãos de maior extensão em tecidos e que as modificações apresentadas pela ação do tempo pode predispor o idoso a risco a saúde e afecções crônicas. A pele íntegra estabelece uma conexão física e tátil do corpo com o meio ambiente, ao mesmo tempo em que estabelece uma função protetora de determinados órgãos e manutenção da temperatura corporal. A manutenção e restabelecimento da integridade desse sistema, no entanto fazem parte de ações de promoção, prevenção, reabilitação e cura, tornando-se cuidados essenciais durante o processo de envelhecimento. ${ }^{2}$

Na população idosa, a incontinência urinária é considerada uma das mais importantes e recorrentes síndromes geriátricas e que ocasiona sérios danos à integridade da pele. Estima-se que acometa cerca de 30\% dos idosos que vivem na comunidade, de 40\% a 70\% dos idosos hospitalizados e 50\% dos idosos que vivem em instituições de longa permanência para idosos (ILPI). ${ }^{3}$

A incontinência urinária tem impacto negativo sobre a vida das pessoas, pois as predispõe às infecções perineais, genitais, do trato urinário e problemas de pele, interrompe o sono, compromete o convívio social, além de ser um dos grandes fatores de risco para quedas na população. ${ }^{4}$ la identificación, la diferenciación y la gestión de la DAI.

Palabras clave: Dermatitis; Incontinencia urinaria/fecal; Tercera edad; Enfermería.

Uma variedade de termos tem sido utilizada para descrever lesões de pele associadas à incontinência, tais como: dermatite perineal, erupção cutânea por uso de fralda, dermatite irritativa de fraldas, dermatite amoniacal, maceração por umidade, dermatite de contato entre outros. ${ }^{5}$ Em 2007, após um encontro entre um grupo de enfermeiros especialistas, foi padronizada a expressão dermatite associada à incontinência (DAI), sendo publicado o $1^{\circ}$ Consenso no Journal of Wound Ostomy \& Continence Nurses (JWOCN), da Sociedade Norte Americana de Enfermeiros Estomaterapeutas. ${ }^{5}$ Para fins deste estudo, esta será a terminologia utilizada.

A DAI é uma manifestação clínica de lesões de pele associadas à umidade, comum em pacientes com incontinência fecal e/ou urinária. É uma inflamação da pele na região perineal, perigenital, perianal e adjacências, provenientes do contato com urina ou fezes. São lesões caracterizadas por erupções cutâneas, erosão da epiderme e aparência macerada. ${ }^{5}$

A prevalência da DAI foi apontada como responsável por 7\% das lesões de pele em pacientes incontinentes internados em casa de repouso, 5,6 50\% das lesões nesta mesma clientela em pacientes com incontinência fecal, $^{5-7} 42 \%$ em adultos incontinentes e hospitaliza$\operatorname{dos}^{5-8}$ e $83 \%$ dos pacientes incontinentes internados em unidades de cuidados intensivos..$^{5-9}$

Alguns estudos indicam que para desenvolver DAI é necessária a presença de irritantes em contato com a pele assim como a duração e a frequência de exposição a esses componentes. $\mathrm{O}$ pH alcalino dos pacientes com incontinência dupla, ou seja, com incontinência urinária e fecal é o responsável pela ativação de lipases e proteases as quais quebram proteínas e contribuem para erosão da epiderme. A hiper-hidratação e a maceração do tecido, elevação da temperatura na região devido ao uso de fraldas, penetração dos irritantes internos (excreções) e externos (produtos), fricção, dentre outros fatores também contribuem para o aparecimento e agravamento dessa situação descrita. ${ }^{5}$

Esta dermatite causa desconforto e dor, que pode ser comparada à dor da queimadura. A dor provocada tem sido relacionada com aumento do risco de úlcera por pressão (UP) devido à limitação imposta à mobilização na tentativa do paciente se proteger. Estudos têm apontado uma tolerância menor à fricção e à pressão nesta 
população, ocasionando maior risco de ulceração. ${ }^{5}$

Sem dúvida, os profissionais da área de saúde têm pela frente um grande desafio, pois verifica-se uma necessidade latente de investimentos em capacitação de recursos humanos que propiciem uma assistência adequada a essa clientela. ${ }^{5}$ Acrescenta-se o fato de que o envelhecimento e a institucionalização aumenta o risco da integridade da pele prejudicada, o que torna ainda mais necessário que os enfermeiros façam um diagnóstico mais precoce possível com vistas a implementação de ações que contribuam para a melhoria do cuidado..$^{10}$ Diante dessa realidade, o presente trabalho tem por objetivo analisar produções científicas que abordam a DAI na população idosa considerando suas características, prevenção e tratamento.

\section{Metodologia}

Trata-se de uma pesquisa bibliográfica, utilizandose o método revisão integrativa em que foram reunidos os resultados de pesquisas sobre as DAIs na população idosa. Este método foi escolhido por permitir a busca, avaliação crítica e síntese das evidências do assunto abordado, ${ }^{11}$ proporcionando, ainda, a incorporação da aplicabilidade dos resultados com base na prática. ${ }^{12}$

O levantamento dos dados ocorreu por meio da busca de artigos disponíveis sobre o assunto nas bases da Biblioteca Virtual de Saúde (BVS) pelo fato de esta compreender a literatura relativa às Ciências da Saúde que congrega bases de dados de vários países, sendo administrada pela Organização Mundial da Saúde e por ser uma das mais visitadas por profissionais da área de saúde. Utilizaram-se como descritores as associações: 1) dermatite, incontinência urinária/fecal, idosos. Para ampliar a busca optou-se por um segundo grupo de associações: 2) dermatite, incontinência, idosos, enfermagem. No primeiro grupo foram encontrados 8 artigos e no segundo, 21 artigos, porém apenas $1 \mathrm{em}$ português. Assim, percebeu-se uma carência de publicações nacionais sobre o tema nessas bases de dados. Optou-se por analisar as produções nos últimos 10 anos, ou seja, do ano de 2006 até 2015. Posteriormente, após leitura atenta, foram selecionados 8 artigos do primeiro grupo e 13 do segundo, totalizando 21 artigos que possuíam relação direta com o tema. Destes, apenas 15 estavam disponíveis em texto completo, sendo 6 deles acessados pela Biblioteca da Faculdade de Enfermagem da UERJ por meio do COMUT (Programa de Comutação Bibliográfica), que permite o acesso a cópias de documentos técnico-científicos disponíveis nos acervos das principais bibliotecas brasileiras e em serviços de informação internacionais e os demais acessados por meio do Portal de Periódico CAPES. Outros textos nacionais publicados em revistas e livros foram utilizados para embasamento bibliográfico e análise dos resultados.

As ideias centrais foram analisadas utilizando a técnica de análise temática de conteúdo, ${ }^{13}$ das quais emergiram as seguintes categorias: DAI como um problema de saúde social e hospitalar e medidas de prevenção e tratamento hospitalar da DAI- um cuidado de enfermagem.

\section{Resultados e discussão}

$\mathrm{Na}$ caracterização das produções selecionadas que abordam a DAI em idosos, quanto ao ano de publicação na BVS (Tabela 1), foi possível observar no período de 2005 a 2008 apenas 3 publicações, ${ }^{14-16}$ enquanto nos períodos de 2009 a 2016 observou-se um crescente número de publicações sobre o assunto, sendo analisadas 12 publicações. ${ }^{17-28}$

A partir de 2007, com a divulgação do $1^{\circ}$ Consenso, e em 2011, com a publicação do $2^{\circ}$ Consenso pela Sociedade Norte-Americana de Enfermeiros Estomaterapeutas, observa-se um aumento no número de estudos sobre a DAI, acreditando-se repousar neste fato a escassez de publicações sobre o assunto neste primeiro período do estudo.

Os artigos analisados procuraram demonstrar as diferentes formas de apresentação da DAI, os fatores predisponentes, a incidência e prevalência entre a população idosa, assim como o impacto social ocasionado em decorrência desta manifestação, protocolos e medidas de prevenção e tratamento.

A partir das ideias principais que emanaram dos artigos selecionados destacaram-se duas categorias a serem discutidas conforme tabelas abaixo (Tabelas 2 e 3):

Primeira categoria: DAI como um problema social e hospitalar.

Em relação à primeira categoria, diversos auto-

Tabela 1. Distribuição dos artigos sobre dermatite associada à incontinência segundo o ano de publicação.

\begin{tabular}{c|c|c|}
\hline Ano de Publicação & $\mathbf{n}$ & $\%$ \\
\hline 2005 a 2008 & 03 & 20 \\
\hline 2009 a 2012 & 08 & 53 \\
\hline 2013 a 2016 & 04 & 27 \\
\hline Total & 15 & 100 \\
\hline
\end{tabular}




\section{Artigo original}

res descrevem a DAI como um processo inflamatório $^{14,15,18,22,25,27,28}$ com características peculiares como eritema, ${ }^{17-20,23,26-28}$ erupções, ${ }^{18}$ vesículas, ${ }^{18,26,27}$ maceração, ${ }^{17,19,28}$ dor, $, 4,18,26$ edema, ${ }^{26,27}$ perda da camada superficial da pele. ${ }^{14}$ Outro autor aponta, além das características citadas, uma área demarcada em "W" como abrangente em região delimitada pelo uso de fralda: superfície convexa de glúteos, coxas, parte inferior do abdômen, púbis e períneo. $^{30}$

Como fatores predisponentes na ocorrência da DAI, indica-se a presença de fezes e urina em contato contínuo com a pele, ocasionando excesso de umidade, $14,15,17,18,20,24,25,27$ fatores mecânicos como fricção e cisalhamento, ${ }^{17,19,24,25,27}$ alteração do $\mathrm{pH}$ do estrato córneo, ${ }^{15,17,19,24,25,27}$ colonização por micro-organismos. . $^{24,25,27}$ Acrescenta-se a questão do envelhecimento com todas as comorbidades associadas: cognição diminuída, incontinências, demência e outros, ${ }^{15,20,24,25,27}$ como a utilização de produtos não adequados para a higienização ${ }^{24,26}$ e o uso de fraldas. ${ }^{26}$

Tabela 2. Categoria I: dermatite associada à incontinência como problema de saúde social e hospitalar.

\begin{tabular}{l|c|c|}
\hline \multicolumn{1}{|l}{ Unidades de significação } & $\mathbf{n}$ & $\%$ \\
\hline $\begin{array}{l}\text { Conceitos e características de identificação } \\
\text { da DAI* }\end{array}$ & 11 & 73,3 \\
\hline Fatores predisponentes da DAI & 10 & 66,7 \\
\hline Incidência, prevalência e impacto social & 10 & 66,7 \\
\hline Diagnóstico diferencial & 2 & 13,3 \\
\hline
\end{tabular}

Tabela 3. Categoria II: prevenção e tratamento da dermatite associada à incontinência.

\begin{tabular}{|c|c|c|}
\hline Unidades de significação & $\mathbf{n}$ & $5 \%$ \\
\hline $\begin{array}{l}\text { Medidas de prevenção: } \\
\text { Higienização }\end{array}$ & 9 & 60,0 \\
\hline Hidratantes e protetores & 8 & 53,3 \\
\hline Tecnologia e instrumentos/dispositivos & 7 & 46,7 \\
\hline Protocolos e ferramentas & 8 & 53,3 \\
\hline Observação e avaliação & 4 & 40,0 \\
\hline $\begin{array}{l}\text { Medidas de tratamento: } \\
\text { Infecção associada à DAI }\end{array}$ & 2 & 13,3 \\
\hline Incontinência associada à DAl & 3 & 20,0 \\
\hline
\end{tabular}

A alta incidência e prevalência de incontinência urinária e fecal na população em estudo, ${ }^{15-16,26}$ sendo a urinária a de maior predominância, ${ }^{17}$ são sinalizadas como questão epidemiológica. As mulheres são mais vulneráveis do que os homens, ${ }^{15,25}$ embora esta prevalência seja subestimada devido à ausência de instrumentos de validação. ${ }^{17}$

Além dos altos custos com o tratamento da DAI, 16,20,22,26 têm-se o atendimento de má qualidade, a baixa qualidade de vida, ${ }^{22}$ além do agravamento do estado geral do paciente, como piora ou predisposição a UP e infecções. ${ }^{16-20,24,25}$ Ao gerar implicações negativas nos âmbitos social, emocional e econômico para o paciente e seus familiares, essa situação vem justificando a atenção dispensada pelo cuidado de enfermagem ${ }^{29}$ e a necessidade de se estabelecerem protocolos para a incontinência com produtos de cuidados econômicos e eficazes. ${ }^{24}$

Evidencia-se a necessidade de uma abordagem de enfermagem direcionada para diferenciação da DAI e UP com utilização de tecnologias de cuidado apropriadas e diferenciação dos sinais apresentados, para o devido tratamento e melhoria das condições de saúde do indivíduo. 17,25,27,28

\section{Segunda categoria: medidas de prevenção e tratamento da DAI - um cuidado de enfermagem}

Em relação à segunda categoria, nota-se um destaque para a questão da higienização e manutenção da pele sem resíduos de fezes e urina, ${ }^{15,17,21}$ assim como de soluções antissépticas ${ }^{19}$ e lenços umedecidos sem álcool foram mencionados. ${ }^{20,26}$ Acrescenta-se o uso de hidratantes e protetores visando criar uma barreira protetora da pele. ${ }^{14-16,24,25}$ Citam-se produtos à base de óxido de zinco, 14,18,21,26-28 petrolatum, ${ }^{18,26-28}$ aloe vera, ${ }^{14,26}$ dimeticona, ${ }^{18,24,26,27}$ gel hidrocoloide e outros, tais como as películas protetoras, ${ }^{14,24,26,27}$ Não se observa eficácia de um produto sobre outro, fato também observado por outros autores. ${ }^{25,30} \mathrm{O}$ que parece fazer diferença seria uma avaliação normatizada, cuidados individuais implementados e os profissionais envolvidos no cuidado. ${ }^{25,26,27}$

A utilização de dispositivos para contenção de fezes e urina também é apontado como fator primordial na abordagem a pacientes com DAI, pois visam manter a pele livre do contato direto com irritantes provenientes das eliminações. ${ }^{15-17,20,21,23,26}$ A utilização de fralda de melhor qualidade também foi mencionada. ${ }^{26}$

Faz-se referência ao uso de protocolos e ferramentas descrevendo um regime de cuidado com a pele, estraté- 
gias para promoção da continência e abordagem para resolução dos problemas. ${ }^{15-17-20,24,26-28}$ Destaca-se a importância de uma avaliação, observação e documentação para prevenir os riscos da DAI, as reações aos produtos e dispositivos utilizados..$^{15,17,26,28}$

As medidas de tratamento, no entanto, focalizamse no tratamento da incontinência que predispõe a DAI ${ }^{15,17,18}$ e da infecção proveniente da mesma. ${ }^{15,18}$ Para o primeiro sugere-se a utilização de dietas, exercícios do assoalho pélvico com foco na contenção de fezes e urina. ${ }^{17}$ Já sobre o tratamento das infecções provenientes da DAI observa-se a utilização de cremes antifúngicos e corticosteroide por curto prazo com foco na erradicação da infecção cutânea. Em casos mais graves, sugere-se o uso de Metronidazol 400mg VO. ${ }^{15}$ Porém, ainda encontram-se divergências sobre o tratamento correto da DAI. ${ }^{30}$

\section{Conclusão}

Os artigos da literatura que foram analisados apresentam recomendações para prevenção, higienização, hidratação, proteção da pele e tratamento da DAI. No entanto, a prevenção e o tratamento da DAI é ainda um grande desafio para os enfermeiros estomaterapeutas tendo em vista a alta incidência, em especial na clientela idosa, e os inúmeros produtos disponibilizados no mercado.

Observa-se a importância de um acompanhamento individualizado e minucioso com registros adequados de forma a facilitar a identificação, a diferenciação e o manejo da DAI. Faz-se necessário a criação de um protocolo individualizado de cuidados com a pele visando à diminuição da DAI, e consequentemente, um menor índice de complicações, tais como UP e infecções.

A partir desses cuidados, almeja-se a implementação de cuidados mais seguros e de qualidade à população idosa mais vulnerável, assim como a diminuição dos enormes gastos com o manejo desta comorbidade ocasionando menor impacto social e hospitalar.

\section{Referências}

1. Instituto Brasileiro de Geografia e Estatística (IBGE). Indicadores sociodemográficos e de saúde no Brasil. 2009. Disponível em: http://www.ibge.gov.br/home/estatistica/populacao/ indic_sociosaude/2009/indicsaude.pdf. Acesso em $14 \mathrm{de}$ dez de 2013.

2. Mendes NPN, Tourinho FSV. Enfermagem e a integridade da pele, da mucosa e dos anexos do idoso hospitalizado. In: Gonçalves LHT, Tourinho FSV (orgs). Enfermagem no cuidado ao idoso hospitalizado. Barueri: Manole; 2012.

3. Silva VA, D'Elboux MJ. Atuação do enfermeiro no manejo da incontinência urinária no idoso: uma revisão integrativa. Rev
Esc Enferm. USP 2012; 46 (5): 1221-6.

4. Chaimowicz F. e cols. Saúde do idoso. 2 ed. Belo Horizonte: Nescon UFMG; 2013. 167 p.

5. Chimentão DMN, Domansky, RC. Dermatite Associada à Incontinência. In: Borges EL, Domansky RC. Manual para prevenção de lesões de pele: recomendações baseadas em evidências. Rio de janeiro: Editora Rubio; 2012.

6. Blizz DZ, Savik K, Harms S, et al. Prevalence and correlates of perineal dermatitis in nursing home residents. Nurs Res. 2006;55(4):243-51.

7. Blizz DZ, Lowry A, Whitebird R, et al. Absorbent product use and incontinence associated dermatitis in community-living persons with fecal incontinence. Abstracts of the 38th Annual Meeting of the International Continence society; October 2008; Cairo, Egypt. Disponível em: https://www.icsoffice.org/Abstracts/Publish/46/000427.pdf. Acesso em 28 de novembro de 2011

8. Driver DS. Perineal dermatitis in critical care patients. Crit Care Nurse. 2007; 27(4):42-6.

9. Peterson KJ, BlizzDz, Nelson C, et al. Practices of nurses and nursing assistants in preventing incontinence dermatitis in acutely/critically ill patients (Abstract). Am J Crit Care. 2006;15(3);325.

10. Rosa NM, Inoue KC, Silvino MCS, et al. Tratamento da dermatite associada à incontinência em idosos institucionalizados: Revisão integrativa. Rev Rene. 2013;14(4):1031-40.

11. Mendes K, Dal S, Silveira RCCP, et al. Revisão integrativa: método de pesquisa para a incorporação de evidências na saúde e na enfermagem. Texto \& Contexto Enfermagem. Out/ dez 2008;17(4):758-764.

12. Souza MT, Silva MD, Carvalho R. Revisão integrativa: o que é e como fazer. Einstein São Paulo. Jan/Mar 2010:8(1):102-6.

13. Bardin L. Análise de Conteúdo. Lisboa: Edições 70;2011.

14. Bliss DZ, Zehrer C, Zavik K, et al. Incontinence-associated skin damage in nursing home residents: A secundary analysis of a prospective, multicenter study. Ostomy wound manage. 2006;46-55.

15. Nazarko L. Mananging a common dermatological problem: incontinence dermatitis. British Journal of Community Nursing. Aug. 2007;12(8):358-63.

16. Al-samarai NR, Uman GC, Al-samaraiTN, et al. Introducing a new continence management system of nursing home residents. J Am Med Dir Assoc. 2007;8(4):253-61.

17. Beeckman D, Woodward S, Gray M. Incontinence-associated dermatitis: step-by-step prevention and treatment. $\mathrm{Br} \mathrm{J}$ Community Nurs. 2011;16(8):382-9.

18. Beeckman D, Verhaeghe S, Defloor T, et al. 3-in-1 perineal pare washcloth impregnated with dimethicone $3 \%$ versus water and $\mathrm{pH}$ neutral soap to prevent and treat Incontinence-associated dermatitis. A randomized, controlled clinical trial. Journal Wound Ostomy Continence Nurs. Aug 2011;38(6):627-4.

19. Bliss DZ, Savik K, Thorson MAL. Incontinence-associated dermatitis in critically ill adults: time to development, severity and risk factors. J Wound Ostomy Continence Nurs. July/August, 2011;38(4):433-45.

20. Denat $Y, K$ Khorshid $L$. The effect of 2 different care products on incontinence-associated dermatitis in patients with fecal incontinence. J Wound Ostomy Continence Nurs. 2011;38(2):171-6.

21. PaleseA, CarnielG. The effects of a multi-intervention incontinence care program on clinical, economic, and environmental outcomes Journal Wound Ostomy Continence Nurse. March/ april 2011;38(2):177-183.

22. Seers K, Cox K, Crichton NJ. FIRE: Facilitating implementa- 


\section{Artigo original}

tion of research evidence - a study protocol. Implementation Science. 2012;7-25.

23. Sugama J, Sanada H, Shigeta Y. Efficaccy of an improved absorvent pad on incontinence-associated dermatitis in older women: cluster randomized controlled trial. BMC Geriatrics. 2012;12-22.

24. Brunner M, Droegemueller C, Rivers S, et al. Prevention of Incontinence-Related Skin Breakdown for Acute and Critical Care Patients: Comparison of Two Products .Urologic Nursing. July-August 2012;32(4):214-19.

25. Corcoran E, Woodward S. Incontinence-associated dermatitis in the elderly: treatment options. British Journal of Nursing. 2013;22(8):450-457.

26. Rosa MN, Inoue KC, Silvino MCS, et al. Tratamento da derma- tite associada à incontinência em idosos institucionalizados: revisão integrativa. Rev Rene. 2013;14(4):1031-40.

27. Holroyd S, Graham K. Prevention and management of incontinence associated dermatitis using a barrier cream. $\mathrm{Br} J$ Community Nurs. Suppl Wound Care. Dec 2014:32-38.

28. Jacobson TM, Wright T. Improving Quality by Taking Aim at Incontinence-Associated Dermatitis in Hospitalized Adults. Med Sung Nurs. May-Jun 2015;24(3):151-7.

29. Silva APM, Santos VCG. Prevalência da incontinência urinária em adultos e idosos hospitalizados. Revista Esc Enfermagem USP. 2005;39(1):36-45.

30. Ribeiro JU, Borges EL. Revisão integrativa do tratamento de dermatite associada à incontinência. Revista Estima. 2010; 8(1):30-39. 